IAU Colloquium 164: Radio Emission from Galactic and Extragalactic Compact Sources

ASP Conference Series, Vol. 144, 1998

J. A. Zensus, G. B. Taylor, \& J.M. Wrobel (eds.)

\title{
A VLBI Survey of 461 Southern Flat Spectrum Sources
}

\author{
E. A. King
}

Australia Telescope National Facility, Canberra, Australia

J. E. J. Lovell

Institute of Space and Astronautical Science, Japan

S. P. Ellingsen, M. E. Costa, P. M. McCulloch

University of Tasmania, Hobart, Australia

J. E. Reynolds

Australia Telescope National Facility, Sydney, Australia

D. L. Jauncey

Australia Telescope National Facility, Canberra, Australia

Abstract. We are undertaking a 1-baseline "cuts" VLBI survey of 461 flat spectrum radio sources selected from the Parkes Catalogue.

Comprehensive surveys provide an essential foundation for statistical studies of cosmological theories and the properties of radio-sources at large. Additionally, surveys lead to the discovery of key objects, such as gravitational lenses, which have the potential to enable us to address many fundamental astrophysical problems, including a determination of the Hubble constant and other cosmological parameters.

We are undertaking a series of "cuts" VLBI observations of a complete sample of 461 Parkes flat-spectrum sources using the University of Tasmania's Mt Pleasant $26 \mathrm{~m}$ antenna and either the ATNF's 22m Mopra antenna or one of the $34 \mathrm{~m}$ or $70 \mathrm{~m}$ antennas at JPL's Tidbinbilla tracking station. These observations, at 2.3 and $8.4 \mathrm{GHz}$, are extending an earlier ATCA search for gravitational lenses (Lovell, in preparation, 1997) by another two orders of magnitude in angular resolution.

The original sample was selected from the Parkes Catalogue according to the following criteria: (1) $2.7 \mathrm{GHz}$ flux density $\geq 0.34 \mathrm{Jy}$, (2) 2.7 to $5.0 \mathrm{GHz}$ spectral index $\alpha \geq-0.5\left(S \propto \nu^{\alpha}\right)$ and (3) B1950 declination $\leq-20^{\circ}$. This yields a sample of 461 objects. The distribution of these sources on the southern sky is shown in Figure 1.

We are undertaking this survey in two phases. In Phase 1, which is essentially a finding survey, we observed each of the sources in the sample on a single-baseline at $2.3 \mathrm{GHz}$ with three widely separated "cuts". This enables us to efficiently determine which objects are detectable at high resolution whilst providing a degree of robustness against non-detection due to orientation effects. In Phase 2, we will carry out more detailed single-baseline followup observations of those objects that show evidence (from Phase 1) of structure more complex than a single component. These observations will be made at both 2.3 and $8.4 \mathrm{GHz}$ to provide spectral index filtering to distinguish between core-jet objects and candidate lenses. We will fit image plane models to these data to determine the basic source structures. Phase 1 is now essentially complete, the data having all been correlated and examined for evidence of source structure. Phase 2 observations are scheduled for June 1997. 


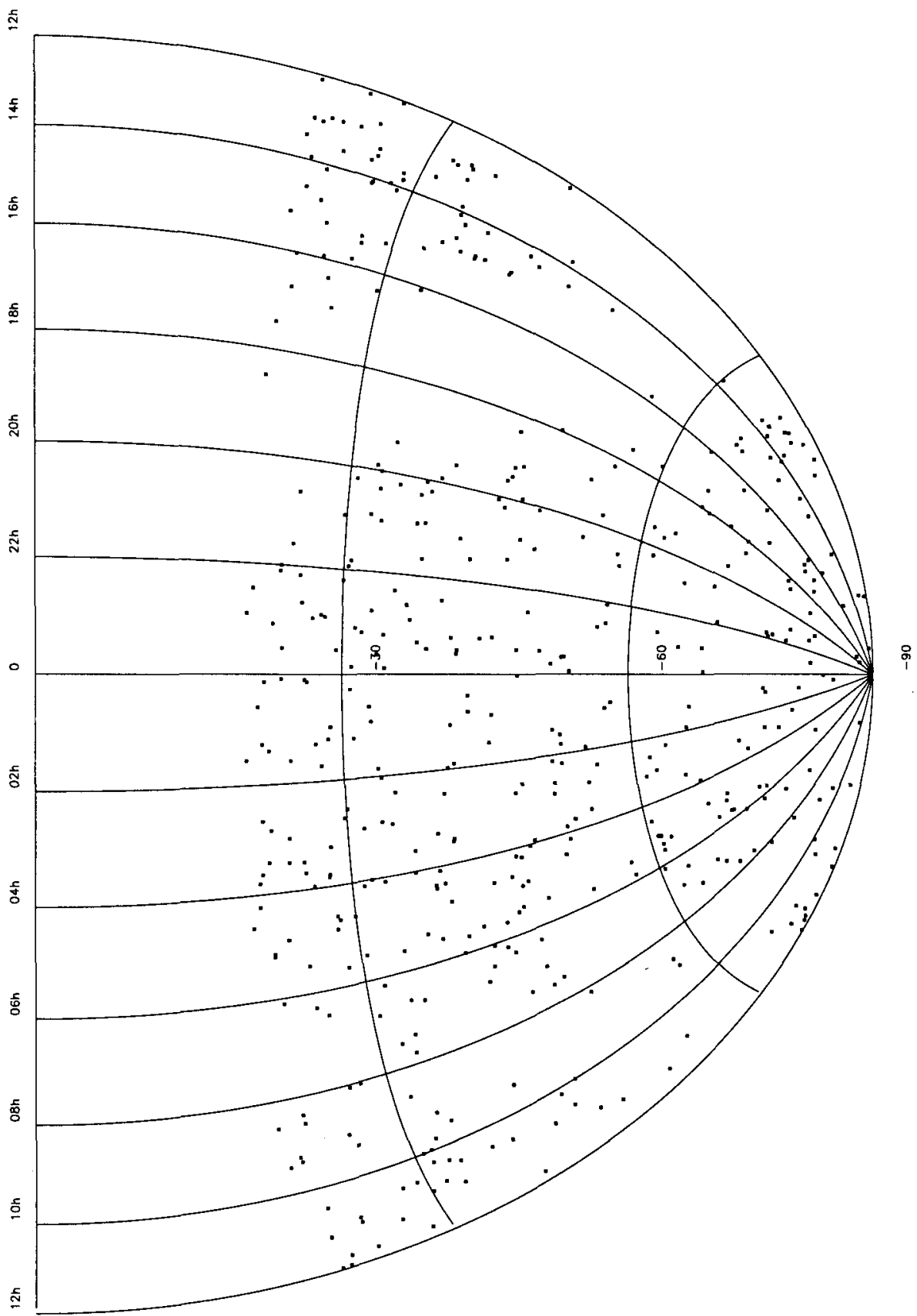

Figure 1. Distribution of the sample objects on the Southern sky. 